\title{
COMPLEXES OF COUSIN TYPE AND MODULES OF GENERALIZED FRACTIONS
}

\author{
SANG-CHO CHUNG
}

\section{Introduction}

Let $\mathbf{R}$ be a commutative (Noetherian) ring, $\mathbf{M}$ an $\mathbf{R}$-module and let $\mathscr{F}=$ $\left(\mathbf{F}_{i}\right)_{i \geq 0}$ be a filtration of $\operatorname{Spec}(\mathbf{R})$ which admits $\mathbf{M}$.

A complex of $\mathbf{R}$-modules is said to be of Cousin type if it satisfies the four conditions of ([GO], 3.2) which are reproduced below (Definition (1.5)). In ([RSZ], 3.4), Riley, Sharp and Zakeri proved that the complex, which is constructed from a chain of special triangular subsets defined in terms of $\mathscr{F}$ (Example (1.3)(3)), is of Cousin type for $\mathbf{M}$ with respect to $\mathscr{F}$ (Corollary (3.5)(2)). Gibson and O'carroll ([GO], 3.6) showed that the complex, which is obtained by means of a chain $\mathcal{U}=\left(\mathbf{U}_{i}\right)_{i \geq 1}$ of saturated triangular subsets and the filtration $\mathscr{G}=\left(\mathbf{G}_{i}\right)_{i \geq 0}$ induced by $\mathcal{U}$ and $\mathbf{M}$, is of Cousin type for $\mathbf{M}$ with respect to $\mathscr{G}$ (Corollary (3.5)(3)).

The purpose of this paper is to show that, when the complex is defined by a chain of triangular subsets, one can give a simpler criterion, consisting of only two conditions, for being of Cousin type (Theorem (3.1) and Corollary (3.2)). In fact, we prove that, for every complex induced by a chain of triangular subsets, the first and the second conditions of the definition of Cousin type hold (Remark (2.5)).

In ([RSZ], 3.3), Riley, Sharp and Zakeri proved that every complex of Cousin type for $\mathbf{M}$ with respect to $\mathscr{F}$ is isomorphic to the Cousin complex. Hence when we investigate the structure of a complex of Cousin type, it is useful to study the complex $\mathbf{C}(\mathcal{U}, \mathbf{M})$ of Cousin type which is constructed from special modules of generalized fractions (Corollary (3.5)) whose properties are well known.

We also get a refinement of the Exactness theorem ([SZ2], 3.3 and [O], 3.1) in our Proposition (2.13).

We wish to thank Prof. H. Matsumura for his continual and stimulating interest in this work.

Received October 28, 1993. 


\section{Preliminaries}

Throughout this paper, $\mathbf{R}$ is a commutative ring with identity and $\mathbf{M}$ is an $\mathbf{R}$-module. We use ${ }^{T}$ to denote matrix transpose and $\mathbf{D}_{n}(\mathbf{R})$ to denote the set of all $n \times n$ lower triangular matrices over $\mathbf{R}$. For $\mathbf{H} \in \mathbf{D}_{n}(\mathbf{R}),|\mathbf{H}|$ denotes the determinant of $\mathbf{H}$. $\mathbf{N}$ denotes the set of positive integers.

Definition (1.1) ([SZ1], 2.1). Let $n$ be a positive integer. A non-empty subset $\mathbf{U}_{n}$ of $\mathbf{R}^{n}$ is said to be triangular if

( i ) whenever $\left(a_{1}, \ldots, a_{n}\right) \in \mathbf{U}_{n}$, then $\left(a_{1}^{\alpha_{1}}, \ldots, a_{n}^{\alpha_{n}}\right) \in \mathbf{U}_{n}$ for all choices of positive integers $\alpha_{1}, \ldots, \alpha_{n}$; and

(ii) whenever $\left(a_{1}, \ldots, a_{n}\right)$ and $\left(b_{1}, \ldots, b_{n}\right) \in \mathbf{U}_{n}$, then there exist $\left(c_{1}, \ldots, c_{n}\right) \in \mathbf{U}_{n}$ and $\mathbf{H}, \mathbf{K} \in \mathbf{D}_{n}(\mathbf{R})$ such that $\mathbf{H}\left[a_{1} \ldots a_{n}\right]^{T}=\left[c_{1} \ldots c_{1}\right]^{T}=\mathbf{K}\left[b_{1} \ldots b_{n}\right]^{T}$.

Definition (1.2) ([S4], 1.1 and 1.2). Let $\mathbf{R}$ be a ring and $\mathbf{M}$ an $\mathbf{R}$-module. A filtration of $\operatorname{Spec}(\mathbf{R})$ is a descending sequence $\mathscr{F}=\left(\mathbf{F}_{i}\right)_{i \geq 0}$ of subsets of $\operatorname{Spec}(\mathbf{R})$, so that

$$
\operatorname{Spec}(\mathbf{R}) \supset \mathbf{F}_{0} \supset \mathbf{F}_{1} \supset \cdots \supset \mathbf{F}_{\imath} \supset \mathbf{F}_{i+1} \supset \cdots,
$$

with the property that, for each $i \geq 0$, each member of $\mathbf{F}_{i} \backslash \mathbf{F}_{i+1}$ is a minimal member of $\mathbf{F}_{\imath}$ with respect to inclusion. We then set $\partial \mathbf{F}_{\imath}=\mathbf{F}_{i} \backslash \mathbf{F}_{\imath+1}$. We say that the filtration $\mathscr{F}$ admits an $\mathbf{R}$-module $\mathbf{M}$ if $\operatorname{Supp}(\mathbf{M}) \subset \mathbf{F}_{0}$. Let $\mathscr{F}_{M}=\left(\mathbf{F}_{\mathbf{M}_{\imath}}\right)_{\imath \geq 0}$ be the $\mathbf{M}$-height filtration of $\operatorname{Spec}(\mathbf{R})$, i.e., $\mathbf{F}_{\mathbf{M} i}=\left\{\mathfrak{p} \in \operatorname{Supp}(\mathbf{M}): \mathrm{ht}_{\mathbf{M}} \mathfrak{p} \geq i\right\}$.

We say that a sequence of elements $a_{1}, \ldots, a_{n}$ of $\mathbf{R}$ is a poor $\mathbf{M}$-sequence if $a_{i}$ is not a zerodivisor on $\mathbf{M} /\left(a_{1}, \ldots, a_{i-1}\right) \mathbf{M}$ for each $i=1, \ldots, n$; it is an $\mathbf{M}$-sequence if, in addition, $\mathbf{M} \neq\left(a_{1}, \ldots, a_{n}\right) \mathbf{M}$.

Example (1.3). Let $\mathbf{R}$ be a Noetherian ring. Then the following five non-empty sets are triangular subsets of $\mathbf{R}^{n}$.

(1) ([SZ1], 3.10) Let $\mathbf{M}$ be a finitely generated $\mathbf{R}$-module.

$\left(\mathbf{U}_{r}\right)_{n}=\left\{\left(a_{1}, \ldots, a_{n}\right) \in \mathbf{R}^{n}: a_{1}, \ldots, a_{n}\right.$ forms a poor $\mathbf{M}$-sequence $\}$.

(2) (cf. [SZ2], 5.2) Suppose that $\mathbf{M}$ is a finitely generated $\mathbf{R}$-module.

$\left(\mathbf{U}_{h}\right)_{n}=\left\{\left(a_{1}, \ldots, a_{n}\right) \in \mathbf{R}^{n}: \mathrm{ht}_{\mathrm{M}}\left(a_{1}, \ldots, a_{i}\right) \mathbf{R} \geq i \quad(1 \leq i \leq n)\right\}$.

(3) ([RSZ], 2.3) Assume that $\mathbf{M}$ is an $\mathbf{R}$-module such that Ass(M) contains only finitely many minimal members.

$\left(\mathbf{U}_{\bar{h}}\right)_{n}=\left\{\left(a_{1}, \ldots, a_{n}\right) \in \mathbf{R}^{n}:\right.$ for each $i=1, \ldots, n, \quad\left(a_{1}, \ldots, a_{i}\right) \mathbf{R} \not \subset \mathfrak{p}$ for all $\left.\mathfrak{p} \in \partial \mathbf{F}_{i-1} \cap \operatorname{Supp}(\mathbf{M})\right\}$.

(4) ([C], 1.1) Suppose that $\mathbf{M}$ is a finitely generated $\mathbf{R}$-module of dimension $d$. 


$$
\left(\mathbf{U}_{s}\right)_{n}=\left\{\left(a_{1}, \ldots, a_{n}\right) \in \mathbf{R}^{n}: \operatorname{dim} \mathbf{M} /\left(a_{1}, \ldots, a_{i}\right) \mathbf{M}=d-i \quad(1 \leq i \leq n)\right\} .
$$

(5) ([C], 1.2) Suppose that $(\mathbf{R}, \mathbf{m})$ is a local ring and $\mathbf{M}$ is a finitely generated $\mathbf{R}$-module.

$\left(\mathbf{U}_{f}\right)_{n}=\left\{\left(a_{1}, \ldots, a_{n}\right) \in \mathbf{R}^{n}: a_{1}, \ldots, a_{n}\right.$ is an $f$-regular sequence (See [SV], p. 252) with respect to $\mathbf{M}$.

$$
=\left\{\left(a_{1}, \ldots, a_{n}\right) \in \mathbf{R}^{n}: \frac{a_{1}}{1}, \ldots, \frac{a_{n}}{1} \text { in } \mathbf{R}_{\mathfrak{p}} \text { forms an } \mathbf{M}_{\mathfrak{p}}\right. \text {-sequence for all }
$$

$\mathfrak{p} \in \operatorname{Supp}(\mathbf{M}) \backslash\{\mathfrak{m}\}$ such that $\left.\left(a_{1}, \ldots, a_{n}\right) \mathbf{R} \subset \mathfrak{p}\right\}$.

For a given triangular subset $\mathbf{U}_{n}$ of $\mathbf{R}^{n}$, let $\overline{\mathbf{U}}_{n}=\left\{\left(a_{1}, \ldots, a_{i}, 1, \ldots, 1\right) \in \mathbf{R}^{n}\right.$ : for all $i(0 \leq i \leq n), \exists a_{i+1}, \ldots, a_{n} \in \mathbf{R}$ s.t. $\left.\left(a_{1}, \ldots, a_{i}, a_{i+1}, \ldots, a_{n}\right) \in \mathbf{U}_{n}\right\}$. This is a triangular subset of $\mathbf{R}^{n}$ and is called the expansion of $\mathbf{U}_{n}$ ([SZ1], p. 38). Then, by ([SZ1], 3.2), we may assume without loss of the generality that $\mathbf{U}_{n}$ is expanded, i.e., $\mathbf{U}_{n}=\overline{\mathbf{U}}_{n}$, when we consider the module of generalized fractions for $\mathbf{M}$ with respect to $\mathbf{U}_{n}$. So, from now on, we assume that every triangular subset is expanded by means of the expansion of itself.

For a fixed non-negative integer $n, \mathbf{U}_{n+1}^{-n-1} \mathbf{M}$ denotes the module of generalized fractions of $\mathbf{M}$ with respect to $\mathbf{U}_{n+1}$ ([SZ1]). The other notation and terminology about the module of generalized fractions follow ([SZ1]).

Definition (1.4) ([RSZ], p. 52). Let $\mathbf{R}$ be a ring. A family $\boldsymbol{U}=\left(\mathbf{U}_{i}\right)_{i \geq 1}$ is called a chain of triangular subsets on $\mathbf{R}$ if the following conditions are satisfied:

(i) $\mathbf{U}_{i}$ is a triangular subset of $\mathbf{R}^{i}$ for all $i \in \mathbf{N}$;

(ii) (1) $\in \mathbf{U}_{1}$;

(iii) whenever $\left(a_{1}, \ldots, a_{i}\right) \in \mathbf{U}_{i}$ with $i \in \mathbf{N}$, then $\left(a_{1}, \ldots, a_{i}, 1\right) \in \mathbf{U}_{i+1}$; and

(iv) whenever $\left(a_{1}, \ldots, a_{i}\right) \in \mathbf{U}_{\text {, with }} 1<i \in \mathbf{N}$, then $\left(a_{1}, \ldots, a_{i-1}\right) \in \mathbf{U}_{i-1}$.

Each $\mathbf{U}_{i}$ leads to a module of generalized fractions $\mathbf{U}_{i}^{-i} \mathbf{M}$ and we can obtain a complex

$$
0 \stackrel{e^{-1}}{\rightarrow} \mathbf{M} \stackrel{e^{0}}{\rightarrow} \mathbf{U}_{1}^{-1} \mathbf{M} \stackrel{e^{1}}{\rightarrow} \mathbf{U}_{2}^{-2} \mathbf{M} \rightarrow \cdots \rightarrow \mathbf{U}_{i}^{-i} \mathbf{M} \stackrel{e^{i}}{\rightarrow} \mathbf{U}_{i+1}^{-\imath-1} \mathbf{M} \rightarrow \cdots
$$

denoted by $\mathbf{C}(\mathcal{U}, \mathbf{M})$, for which $e^{0}(m)=\frac{m}{(1)}$ for all $m \in \mathbf{M}$ and

$$
e^{i}\left(\frac{x}{\left(a_{1}, \ldots, a_{\imath}\right)}\right)=\frac{x}{\left(a_{1}, \ldots, a_{i}, 1\right)}
$$

for all $i \in \mathbf{N}, x \in \mathbf{M}$ and $\left(a_{1}, \ldots, a_{i}\right) \in \mathbf{U}_{i}$.

$H_{U}^{i}(\mathbf{M})$ denotes the $i$-th cohomology group of $\mathbf{C}(\mathcal{U}, \mathbf{M})$. That is $H_{U}^{i}(\mathbf{M})=$ $\operatorname{Ker} e^{i} / \operatorname{Im} e^{i-1}$. 
Definition (1.5) ([GO], 3.2). Let $\mathbf{R}$ be a Noetherian ring and $\mathbf{M}$ an $\mathbf{R}$-module. Let $\mathscr{F}=\left(\mathbf{F}_{i}\right)_{i \geq 0}$ be a filtration of $\operatorname{Spec}(\mathbf{R})$ that admits $\mathbf{M}$. A complex $\mathbf{X}^{\cdot}=\left\{\mathbf{X}^{i}\right.$ : $i \geq-2\}$ of $\mathbf{R}$-modules and $\mathbf{R}$-homomorphisms is said to be of Cousin type for $\mathbf{M}$ with respect to $\mathscr{F}$ if it has the form

$$
0 \stackrel{d^{-2}}{\rightarrow} \mathbf{M} \stackrel{d^{-1}}{\rightarrow} \mathbf{X}^{0} \stackrel{d^{0}}{\rightarrow} \mathbf{X}^{1} \rightarrow \cdots \rightarrow \mathbf{X}^{i} \stackrel{d^{i}}{\rightarrow} \mathbf{X}^{i+1} \rightarrow \cdots
$$

and satisfies the following, for each $n \in \mathbf{N} \cup\{0\}$,

(i) $\operatorname{Supp}\left(\mathbf{X}^{n}\right) \subset \mathbf{F}_{n}$;

(ii) $\operatorname{Supp}\left(\right.$ Coker $\left.d^{n-2}\right) \subset \mathbf{F}_{n}$;

(iii) $\operatorname{Supp}\left(\operatorname{Ker} d^{n-1} / \operatorname{Im} d^{n-2}\right) \subset \mathbf{F}_{n+1}$; and

(iv) The natural $\mathbf{R}$-homomorphism $\xi\left(\mathbf{X}^{n}\right): \mathbf{X}^{n} \rightarrow \bigoplus_{\mathfrak{p} \in \partial \mathbf{F}_{n}}\left(\mathbf{X}^{n}\right)_{p}$, such that, for $x \in$ $\mathbf{X}^{n}$ and $\mathfrak{p} \in \partial \mathbf{F}_{n}$, the component of $\xi\left(\mathbf{X}^{n}\right)(x)$ in the summand $\left(\mathbf{X}^{n}\right)_{\mathfrak{p}}$ is $x / 1$, is an isomorphism.

Lemma (1.6). Let $\mathbf{R}$ be a ring and $\mathbf{M}$ an $\mathbf{R}$-module. Let $\mathbf{U}_{n}$ be an expanded triangular subset of $\mathbf{R}^{n}$. Let $\left(a_{1}, \ldots, a_{n}\right)$ and $\left(b_{1}, \ldots, b_{n}\right)$ be elements of $\mathbf{U}_{n}$ such that $\mathbf{H}\left[a_{1} \ldots a_{n}\right]^{T}=\left[b_{1} \ldots b_{n}\right]^{T}$ for some $\mathbf{H} \in \mathbf{D}_{n}(\mathbf{R})$. Then we have

(1) $\left([\mathrm{SZ1}], 2.8\right.$ and 3.3(i)) $\frac{m}{\left(a_{1}, \ldots, a_{n}\right)}=\frac{|\mathbf{H}| m}{\left(b_{1}, \ldots, b_{n}\right)}$ and $\frac{a_{n} m}{\left(a_{1}, \ldots, a_{n}\right)}=$ $\frac{m}{\left(a_{1}, \ldots, a_{n-1}, 1\right)}$ in $\mathbf{U}_{n}^{-n} \mathbf{M}$.

(2) ([SZ1, 3.3(ii)] and [SY, 2.2]) If $m \in\left(a_{1}, \ldots, a_{n-1}\right) \mathbf{M}$ then $\frac{m}{\left(a_{1}, \ldots, a_{n}\right)}=0$ in $\mathbf{U}_{n}^{-n} \mathbf{M}$. In particular, if each element of $\mathbf{U}_{n}$ is a poor $\mathbf{M}$-sequence, then the converse holds.

(3) ([SZ2], 5.1 and [SZ3], 2.1) $\operatorname{Ann}_{\mathbf{R}}\left(\frac{m}{\left(a_{1}, \ldots, a_{n}\right)}\right)=\operatorname{Ann}_{\mathbf{R}}\left(\frac{m}{\left(a_{1}, \ldots, a_{n-1}, 1\right)}\right)$.

Lemma (1.7) ([C], 2.4). Let ( $\mathbf{R}, \mathfrak{m})$ be a Noetherian local ring and let $\mathbf{M}$ be a finitely generated $\mathbf{R}$-module of dimension $d$. Let $\left(\mathbf{U}_{s}\right)_{d+1}$ be the expansion of the triangular subset $\left\{\left(a_{1}, \ldots, a_{d}, 1\right) \in \mathbf{R}^{d+1}: \operatorname{dim} \mathbf{M} /\left(a_{1}, \ldots, a_{d}\right) \mathbf{M}=0\right\}$. Let $\left\{x_{1}, \ldots\right.$, $\left.x_{d}\right\}$ be a fixed system of parameters for $\mathbf{M}$. Then we have

$$
\left(\mathbf{U}_{s}\right)_{d+1}^{-d-1} \mathbf{M} \cong \mathbf{U}(x)_{d}[1]^{-d-1} \mathbf{M} \cong \mathbf{H}_{\mathrm{m}}^{d}(\mathbf{M})
$$

where $\mathbf{U}(x)_{d}[1]=\left\{\left(x_{1}^{\alpha_{1}}, \ldots, x_{d}^{\alpha_{d}}, 1\right) \in \mathbf{R}^{n+1}\right.$ : there is $i(0 \leq i \leq d)$ such that $\alpha_{1}, \ldots, \alpha_{i} \in \mathbf{N}$ and $\left.\alpha_{i+1}=\cdots=\alpha_{d}=0\right\}$. 
Lemma (1.8) ([GO], 3.4). Let $\mathbf{R}$ be a ring. For a positive integer $n$, suppose that $\frac{m}{\left(a_{1}, \ldots, a_{n}, 1\right)}=0$ in $\mathbf{U}_{n+1}^{-n-1} \mathbf{M}$. Then there exist $\left(b_{1}, \ldots, b_{n+1}\right) \in \mathbf{U}_{n+1}$ and $\mathbf{H} \in \mathbf{D}_{n}(\mathbf{R})$ such that $\mathbf{H}\left[a_{1} \ldots a_{n}\right]^{T}=\left[b_{1} \ldots b_{n}\right]^{T}$ and $b_{n+1}|\mathbf{H}| m \in\left(b_{1}, \ldots\right.$, $\left.b_{n}\right) \mathbf{M}$.

Lemma (1.9) ([GO], 3.3 and [SY], 2.7). Let $\mathbf{R}$ be a ring and $\mathbf{M}$ an $\mathbf{R}$-module. Let $\mathcal{U}=\left(\mathbf{U}_{i}\right)_{i \geq 1}$ be a chain of triangular subsets on $\mathbf{R}$. Then in $\mathbf{C}(\mathcal{U}, \mathbf{M})$, for all $n \in \mathbf{N}$

$$
\text { Coker } e^{n-1} \cong \mathbf{U}_{n}^{-n} \mathbf{M} / \operatorname{Im} e^{n-1} \cong \mathbf{U}_{n}[1]^{-n-1} \mathbf{M},
$$

where $\mathbf{U}_{n}[1]=\left\{\left(a_{1}, \ldots, a_{n}, 1\right) \in \mathbf{R}^{n+1}:\left(a_{1}, \ldots, a_{n}\right) \in \mathbf{U}_{n}\right\}$.

\section{Associated prime ideals of modules of generalized fractions}

Lemma (2.1). Let $\mathbf{R}$ be a ring and $\mathbf{M}$ an $\mathbf{R}$-module. Fix a positive integer $n$. Let $\mathbf{U}_{n}$ be a triangular subsets of $\mathbf{R}^{n}$. Let $0 \neq \frac{m}{\left(a_{1}, \ldots, a_{n}\right)} \in \mathbf{U}_{n}^{-n} \mathbf{M}$. Then we have, for all $\left(b_{1}, \ldots, b_{n}\right) \in \mathbf{U}_{n}$,

$$
\left(b_{1}, \ldots, b_{n}\right) \mathbf{R} \not \subset\left(0: \frac{m}{\left(a_{1}, \ldots, a_{n}\right)}\right) .
$$

Proof. Suppose that for some $\left(b_{1}, \ldots, b_{n}\right) \in \mathbf{U}_{n}$

$$
\left(b_{1}, \ldots, b_{n}\right) \mathbf{R} \subset\left(0: \frac{m}{\left(a_{1}, \ldots, a_{n}\right)}\right) .
$$

Then by the definition of triangular subset there are $\left(c_{1}, \ldots, c_{n}\right) \in \mathbf{U}_{n}$ and $\mathbf{H}$, $\mathbf{K} \in \mathbf{D}_{n}(\mathbf{R})$ such that $\mathbf{H}\left[a_{1} \ldots a_{n}\right]^{T}=\left[c_{1} \ldots c_{n}\right]^{T}=\mathbf{K}\left[b_{1} \ldots b_{n}\right]^{T}$. Hence we get $\left(c_{1}, \ldots, c_{n}\right) \mathbf{R} \subset\left(b_{1}, \ldots, b_{n}\right) \mathbf{R}$.

On the other hand, by Lemma $(1.6)(1)(3)$ we have

$$
\begin{aligned}
\left(0: \frac{m}{\left(a_{1}, \ldots, a_{n}\right)}\right) & =\left(0: \frac{|\mathbf{H}| m}{\left(c_{1}, \ldots, c_{n}\right)}\right)=\left(0: \frac{|\mathbf{H}| m}{\left(c_{1}, \ldots, c_{n-1}, 1\right)}\right) \\
& \supset\left(b_{1}, \ldots, b_{n}\right) \mathbf{R} \supset\left(c_{1}, \ldots, c_{n}\right) \mathbf{R} .
\end{aligned}
$$

Therefore we have the following contradiction. 


$$
\frac{c_{n}|\mathbf{H}| m}{\left(c_{1}, \ldots, c_{n}\right)}=\frac{|\mathbf{H}| m}{\left(c_{1}, \ldots, c_{n-1}, 1\right)}=0
$$

From now on, we suppose that $\mathbf{U}_{0}[1]^{-1} \mathbf{M}=\mathbf{M}, \mathbf{U}_{0}^{0} \mathbf{M}=\mathbf{M}$ and $n$ is a non-negative integer.

Lemma (2.2). Let $\mathbf{R}$ and $\mathbf{M}$ be as above. Then in $\mathbf{C}(\mathcal{U}, \mathbf{M})$ we have

$$
\operatorname{Supp}\left(\mathbf{U}_{n+1}^{-n-1} \mathbf{M}\right) \subset \operatorname{Supp}\left(\mathbf{U}_{n}[1]^{-n-1} \mathbf{M}\right) \subset \operatorname{Supp}\left(\mathbf{U}_{n}^{-n} \mathbf{M}\right) .
$$

Proof. For the first half, this follows from the following short exact sequence

$$
\begin{aligned}
& 0 \rightarrow \operatorname{Ker} e^{n} / \operatorname{Im} e^{n-1} \rightarrow \mathbf{U}_{n}^{-n} \mathbf{M} / \operatorname{Im} e^{n-1} \rightarrow \mathbf{U}_{n}^{-n} \mathbf{M} / \operatorname{Ker} e^{n} \rightarrow 0, \\
& \mathbf{U}_{n}[1]^{-n-1} \mathbf{M} \quad \operatorname{Im} e^{n}
\end{aligned}
$$

since $\operatorname{Supp}\left(\mathbf{U}_{n+1}^{-n-1} \mathbf{M}\right)=\operatorname{Supp}\left(\operatorname{Im} e^{n}\right)$ by Lemma (1.6)(3).

For the second inclusion, it follows from Lemma (1.9) that

$$
\operatorname{Supp}\left(\mathbf{U}_{n}[1]^{-n-1} \mathbf{M}\right)=\operatorname{Supp}\left(\mathbf{U}_{n}^{-n} \mathbf{M} / \operatorname{Im} e^{n-1}\right) \subset \operatorname{Supp}\left(\mathbf{U}_{n}^{-n} \mathbf{M}\right) .
$$

Example (2.3). In general, $\operatorname{Supp}\left(\mathbf{U}_{n+1}^{-n-1} \mathbf{M}\right) \neq \operatorname{Supp}\left(\mathbf{U}_{u}[1]^{-n-1} \mathbf{M}\right)$. Let $(\mathbf{R}, \mathbf{m})$ be a Noetherian local ring. Suppose that $\mathbf{M}$ is an $f$-module (see [SZ4], 1.8(ii)) of dimension $d$. Then $\operatorname{Supp}\left(\left(\mathbf{U}_{f}\right)_{d}[1]^{-d-1} \mathbf{M}\right)=\operatorname{Supp}\left(\left(\mathbf{U}_{s}\right)_{d+1}^{-d-1} \mathbf{M}\right)=\{\mathrm{m}\}$. But $\operatorname{Supp}\left(\left(\mathbf{U}_{f}\right)_{d+1}^{-d-1} \mathbf{M}\right)=\emptyset$ by $([\mathrm{C}], 2.3)$.

Lemma (2.4). Let $\mathbf{R}$ and $\mathbf{M}$ be as above. Then in $\mathbf{C}(\mathcal{U}, \mathbf{M})$ we have

$$
\operatorname{Supp}\left(\mathbf{U}_{n+1}^{-n-1} \mathbf{M}\right) \subset \operatorname{Supp}\left(\mathbf{U}_{n}[1]^{-n-1} \mathbf{M}\right) \subset \mathbf{F}_{M n} \subset \mathbf{F}_{n} .
$$

Proof. This follows from Lemma (2.2), ([HS], 3.1) and ([C], 2.7).

Remark (2.5). Lemma (2.4) shows that, for every complex $\mathbf{C}(\mathcal{U}, \mathbf{M})$, the first and the second conditions of the definiton of Cousin type hold by Lemma (1.9).

Lemma (2.6). Let $\mathbf{R}$ and $\mathbf{M}$ be as above. Then in $\mathbf{C}(\mathcal{U}, \mathbf{M})$ we have the following.

(1) $\partial \mathbf{F}_{n} \cap \operatorname{Supp}(\mathbf{M})=\left(\cup_{i=0}^{n} \partial \mathbf{F}_{\mathbf{M}_{i}}\right) \cap \partial \mathbf{F}_{n}$.

(2) (cf. [ST], 2.7) $\partial \mathbf{F}_{n} \cap \operatorname{Supp}\left(\mathbf{U}_{n+1}^{-n-1} \mathbf{M}\right) \subset \partial \mathbf{F}_{n} \cap \operatorname{Supp}\left(\mathbf{U}_{n}[1]^{-n-1} \mathbf{M}\right) \subset \partial \mathbf{F}_{n} \cap$ $\partial \mathbf{F}_{\mathrm{M} n}$.

(3) $\partial \mathbf{F}_{n} \cap \partial \mathbf{F}_{\mathrm{M} n}=U_{q \in \partial \mathbf{F}_{n-1} \cap \partial \mathbf{F}_{\mathrm{M}(n-1)}}\left(V(q) \cap \partial \mathbf{F}_{n} \cap \partial \mathbf{F}_{\mathrm{M} n}\right)$. 
Proof. (1) Let $\mathfrak{p} \in \partial \mathbf{F}_{n} \cap \operatorname{Supp}(\mathbf{M}) \backslash \cup_{i=0}^{n} \partial \mathbf{F}_{\mathbf{M} i}$. Hence $\mathrm{ht}_{\mathbf{M}} \mathfrak{p}>n$. Therefore there is $\mathfrak{q} \in \partial \mathbf{F}_{\mathrm{M} n}\left(\subset \mathbf{F}_{n}\right)$ such that $\mathfrak{q} \varsubsetneqq \mathfrak{p}$. That is, $\mathfrak{p}$ is not minimal in $\mathbf{F}_{n}$.

(2) Since $\operatorname{Supp}\left(\mathbf{U}_{n+1}^{-n-1} \mathbf{M}\right) \subset \operatorname{Supp}\left(\mathbf{U}_{n}[1]^{-n-1} \mathbf{M}\right) \subset \mathbf{F}_{\mathbf{M} n}$, we have

$$
\begin{aligned}
\partial \mathbf{F}_{n} \cap \operatorname{Supp}\left(\mathbf{U}_{n+1}^{-n-1} \mathbf{M}\right) & \subset \partial \mathbf{F}_{n} \cap \operatorname{Supp}\left(\mathbf{U}_{n}[1]^{-n-1} \mathbf{M}\right) \subset \partial \mathbf{F}_{n} \cap \operatorname{Supp}(\mathbf{M}) \cap \mathbf{F}_{\mathrm{M} n} \\
& \subset\left(\bigcup_{i=0}^{n} \partial \mathbf{F}_{\mathrm{M} i}\right) \cap \partial \mathbf{F}_{n} \cap \mathbf{F}_{\mathrm{M} n}=\partial \mathbf{F}_{n} \cap \partial \mathbf{F}_{\mathrm{M} n}
\end{aligned}
$$

by (1).

(3) Let $\mathfrak{p} \in \partial \mathbf{F}_{n}$ and $\mathrm{ht}_{\mathrm{M}} \mathfrak{p}=n$. Suppose that $\mathfrak{q} \notin \partial \mathbf{F}_{n-1}$ for some $\mathfrak{q} \in$ $\operatorname{Supp}(\mathbf{M})$ such that ht $\mathrm{M}_{\mathbf{M}} \mathfrak{q}=n-1$ and $\mathfrak{q} \varsubsetneqq \mathfrak{p}$. Hence $\mathfrak{q} \in \mathbf{F}_{n}$, since $\partial \mathbf{F}_{n-1}=\mathbf{F}_{n-1} \backslash$ $\mathbf{F}_{n}$ and $\mathbf{F}_{\mathbf{M}(n-1)} \subset \mathbf{F}_{n-1}$. This contradicts that $\mathfrak{p}$ is a minimal element in $\mathbf{F}_{n}$.

Lemma (2.7). Let $\mathbf{R}$ be a ring and $\mathbf{M}$ an $\mathbf{R}$-molule. Then in $\mathbf{C}(\mathcal{U}, \mathbf{M})$, for each $\frac{m}{\left(a_{1}, \ldots, a_{n}\right)}+\operatorname{Im} e^{n-1} \in H_{U}^{n}(\mathbf{M})$, there are $\left(b_{1}, \ldots, b_{n+1}\right) \in \mathbf{U}_{n+1}$ and $\mathbf{H} \in \mathbf{D}_{n}(\mathbf{R})$ such that $\mathbf{H}\left[a_{1} \ldots a_{n}\right]^{T}=\left[b_{1} \ldots b_{n}\right]^{T}$ and

$$
\left(b_{1}, \ldots, b_{n+1}\right) \mathbf{R} \subset\left(\operatorname{Im} e^{n-1}: \frac{m}{\left(a_{1}, \ldots, a_{n}\right)}\right) .
$$

Proof. Since $\frac{m}{\left(a_{1}, \ldots, a_{n}\right)} \in \operatorname{Ker} e^{n}$, we have $\frac{m}{\left(a_{1}, \ldots, a_{n}, 1\right)}=0$ in $\mathbf{U}_{n+1}^{-n-1} \mathbf{M}$. Hence by Lemma (1.8) there are $\left(b_{1}, \ldots, b_{n+1}\right) \in \mathbf{U}_{n+1}$ and $\mathbf{H} \in \mathbf{D}_{n}(\mathbf{R})$ such that $\mathbf{H}\left[a_{1} \ldots a_{n}\right]^{T}=\left[b_{1} \ldots b_{n}\right]^{T}$ and $b_{n+1}|\mathbf{H}| m \in\left(b_{1}, \ldots, b_{n}\right) \mathbf{M}$. Therefore we have

$$
\left(b_{1}, \ldots, b_{n+1}\right) \mathbf{R} \subset\left(\operatorname{Im} e^{n-1}: \frac{|\mathbf{H}| m}{\left(b_{1}, \ldots, b_{n}\right)}\right)=\left(\operatorname{Im} e^{n-1}: \frac{m}{\left(a_{1}, \ldots, a_{n}\right)}\right) .
$$

Lemma (2.8). Let $\mathbf{R}$ be a ring and $\mathbf{M}$ an $\mathbf{R}$-module. Let $\boldsymbol{U}=\left(\mathbf{U}_{i}\right)_{i \geq 1}$ be a chain of triangular subsets on $\mathbf{R}$. Then in $\mathbf{C}(\mathcal{U}, \mathbf{M})$, for a fixed non-negative integer $n$, we have the following.

(1) $\operatorname{Ass}\left(\mathbf{U}_{n+1}^{-n-1} \mathbf{M}\right) \cap \operatorname{Supp}\left(\mathbf{U}_{n+2+i}^{-n-2-i} \mathbf{M}\right)=\emptyset$ for all $i \geq 0$.

(2) $\operatorname{Ass}\left(\mathbf{U}_{n+1}^{-n-1} \mathbf{M}\right) \cap \operatorname{Supp}\left(\mathbf{U}_{n+1+i}[1]^{-n-2-i} \mathbf{M}\right)=\emptyset$ for all $i \geq 0$.

(3) $\operatorname{Ass}\left(\mathbf{U}_{n+1}^{-n-1} \mathbf{M}\right)=\operatorname{Ass}\left(\operatorname{Im} e^{n}\right)=\operatorname{Ass}\left(\operatorname{Ker} e^{n+1}\right)$.

(4) $\operatorname{Ass}\left(\mathbf{U}_{n+1}^{-n-1} \mathbf{M}\right) \cap \operatorname{Supp}\left(H_{U}^{n+i}(\mathbf{M})\right)=\emptyset$ for all $i \geq 0$.

(5) $\operatorname{Ass}\left(H_{U}^{n}(\mathbf{M})\right) \subset \operatorname{Ass}\left(\mathbf{U}_{n}[1]^{-n-1} \mathbf{M}\right) \subset \operatorname{Ass}\left(H_{U}^{n}(\mathbf{M})\right) \cup \operatorname{Ass}\left(\mathbf{U}_{n+1}^{-n-1} \mathbf{M}\right)$.

(6) If $\mathbf{R}$ is Noetherian, then

$$
\partial \mathbf{F}_{n} \cap \operatorname{Ass}\left(\mathbf{U}_{n}[1]^{-n-1} \mathbf{M}\right)=\left(\partial \mathbf{F}_{n} \cap \operatorname{Ass}\left(H_{U}^{n}(\mathbf{M})\right)\right) \cup\left(\partial \mathbf{F}_{n} \cap \operatorname{Ass}\left(\mathbf{U}_{n+1}^{-n-1} \mathbf{M}\right)\right) .
$$

(7) $\operatorname{Ass}\left(\mathbf{U}_{n}[1]^{-n-1} \mathbf{M}\right) \cap \operatorname{Ass}\left(\mathbf{U}_{n+1}[1]^{-n-2} \mathbf{M}\right) \subset \operatorname{Ass}\left(H_{U}^{n}(\mathbf{M})\right)$. 
Proof. (1) and (2) follow from Lemma (2.1) and Lemma (1.6)(2).

(3) Since $\operatorname{Im} e^{n} \subset \operatorname{Ker} e^{n+1} \subset \mathbf{U}_{n+1}^{-n-1} \mathbf{M}$, this follows from Lemma (1.6)(3).

(4) This follows from Lemma (2.1), Lemma (2.7) and Lemma. (1.6)(2).

(5) The following short exact sequence and (3) complete the proof.

(*)

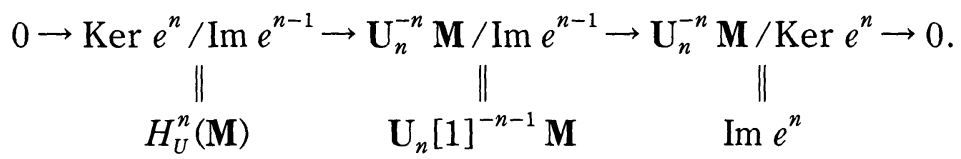

(6) By Lemma (2.4), we have

$$
\partial \mathbf{F}_{n} \cap \operatorname{Supp}\left(\mathbf{U}_{n+1}^{-n-1} \mathbf{M}\right)=\partial \mathbf{F}_{n} \cap \operatorname{Ass}\left(\mathbf{U}_{n+1}^{-n-1} \mathbf{M}\right) \subset \partial \mathbf{F}_{n} \cap \operatorname{Ass}\left(\mathbf{U}_{n}[1]^{-n-1} \mathbf{M}\right)
$$

Hence the assertion follows from (5).

(7) This follows from (1), (4) and (5).

Remark (2.9). If we also change associated prime to weakly associated in the sense of ([B], p. 289 ex. 17), then we can omit the Noetherian condition of Proposition $(2.8)(6)$.

Proposition (2.10). Let $\mathbf{R}$ and $\mathbf{M}$ be as above. Assume that $\mathfrak{p} \in \operatorname{Spec}(\mathbf{R})$. In $\mathbf{C}(\mathcal{U}, \mathbf{M})$, consider the following statements:

(i) For all $\left(a_{1}, \ldots, a_{n+1}\right) \in \mathbf{U}_{n+1},\left(a_{1}, \ldots, a_{n+1}\right) \mathbf{R} \not \subset \mathfrak{p}$;

(ii) $\left(\mathbf{U}_{n+1}^{-n-1} \mathbf{M}\right)_{\mathfrak{p}} \cong\left(\mathbf{U}_{n}[1]^{-n-1} \mathbf{M}\right)_{\mathfrak{p}}$;

(ii') $\left(H_{U}^{n}(\mathbf{M})\right)_{\mathfrak{p}}=0$ and $\left(\mathbf{U}_{n+1}[1]^{-n-2} \mathbf{M}\right)_{\mathfrak{p}}=0$;

(iii) $\left(\mathbf{U}_{n+1}^{-n-1} \mathbf{M}\right)_{\mathfrak{p}} \cong\left(\operatorname{Im} e^{n}\right)_{\mathfrak{p}}$;

(iii') $\left(\mathbf{U}_{n+1}[1]^{-n-2} \mathbf{M}\right)_{\mathfrak{p}}=0$;

(iii") $\left(H_{U}^{n+1}(\mathbf{M})\right)_{\mathfrak{p}}=0$ and $\left(\mathbf{U}_{n+2}^{-n-2} \mathbf{M}\right)_{\mathfrak{p}}=0$;

(iv) $\left(\operatorname{Ker} e^{n+1}\right)_{\mathfrak{p}} \cong\left(\operatorname{Im} e^{n}\right)_{\mathfrak{p}}$;

(iv') $\left(\mathbf{U}_{n+1}[1]^{-n-2} \mathbf{M}\right)_{\mathfrak{p}} \cong\left(\operatorname{Im} e^{n+1}\right)_{\mathfrak{p}}$.

Then we have the following.

(1) (ii) $\Leftrightarrow$ (ii').

(2) (iii) $\Leftrightarrow\left(\right.$ iii' $^{\prime} \Leftrightarrow\left(\right.$ iii $\left.^{\prime \prime}\right)$.

(3) (iv) $\Leftrightarrow$ (iv').

(4) (i) $\Rightarrow$ (ii) $\Rightarrow$ (iii) $\Rightarrow$ (iv). That is, if (i) holds, then

$$
\left(\mathbf{U}_{n+1}^{-n-1} \mathbf{M}\right)_{\mathfrak{p}} \cong\left(\mathbf{U}_{n}[1]^{-n-1} \mathbf{M}\right)_{\mathfrak{p}} \cong\left(\operatorname{Im} e^{n}\right)_{\mathfrak{p}} \cong\left(\operatorname{Ker} e^{n+1}\right)_{\mathfrak{p}} .
$$

(5) If $\mathfrak{p} \in \operatorname{Ass}\left(\mathbf{U}_{n+1}^{-n-1} \mathbf{M}\right)$, then the above four modules are isomorphic.

(6) If $\mathfrak{p} \notin \operatorname{Supp}\left(\mathbf{U}_{n+2}^{-n-2} \mathbf{M}\right)$, then (iv) $\Rightarrow$ (iii). 
Proof. (1) Using the short exact exact sequence $(*)$, we prove as follows. $(\Rightarrow)$ Assume that $\left(\mathbf{U}_{n+1}^{-n-1} \mathbf{M}\right)_{\mathfrak{p}} \cong\left(\mathbf{U}_{n}[1]^{-n-1} \mathbf{M}\right)_{\mathfrak{p}}$. Then, from the following short exact sequence

$$
\begin{gathered}
0 \rightarrow \operatorname{Im} e^{n-1} \rightarrow \mathbf{U}_{n}^{-n} \mathbf{M} \rightarrow \mathbf{U}_{n}^{-n} \mathbf{M} / \operatorname{Im} e^{n-1} \rightarrow 0 \\
\mathbf{U}_{n}[1]^{-n-1} \mathbf{M}
\end{gathered}
$$

we have a commutative diagram with exact rows.

$$
\begin{aligned}
& 0 \rightarrow \underset{\|}{\left(\operatorname{Im} e^{n-1}\right)_{\mathfrak{p}}} \rightarrow\left(\mathbf{U}_{n}^{-n} \mathbf{M}\right)_{\mathfrak{p}} \rightarrow\left(\mathbf{U}_{n}[1]^{-n-1} \mathbf{M}\right)_{\mathfrak{p}} \rightarrow 0 \\
& 0 \rightarrow\left(\operatorname{Im} e^{n-1}\right)_{\mathfrak{p}} \rightarrow\left(\mathbf{U}_{n}^{-n} \mathbf{M}\right)_{\mathfrak{p}} \stackrel{e_{\mathfrak{p}}^{n}}{\rightarrow} \quad\left(\mathbf{U}_{n+1}^{-n-1} \mathbf{M}\right)_{\mathfrak{p}} \quad \rightarrow 0 .
\end{aligned}
$$

Therefore we get

$$
\left(\operatorname{Ker} e^{n}\right)_{p}=\left(\operatorname{Im} e^{n-1}\right)_{p} .
$$

Hence, from the following short exact sequence

$$
\begin{aligned}
& 0 \rightarrow\left(H_{U}^{n}(\mathbf{M})\right)_{\mathfrak{p}} \rightarrow\left(\mathbf{U}_{n}[1]^{-n-1} \mathbf{M}\right)_{\mathfrak{p}} \rightarrow\left(\operatorname{Im} e^{n}\right)_{\mathfrak{p}} \rightarrow 0 \\
& \|\quad\|
\end{aligned}
$$

induced from the short exact sequence $(*)$, we have

$$
\left(\mathbf{U}_{n+1}^{-n-1} \mathbf{M}\right)_{\mathfrak{p}} \cong\left(\mathbf{U}_{n}[1]^{-n-1} \mathbf{M}\right)_{\mathfrak{p}} \cong\left(\operatorname{Im} e^{n}\right)_{\mathfrak{p}}
$$

Therefore from the following short exact sequence

$(* *)$

$$
0 \rightarrow \operatorname{Im} e^{n} \rightarrow \mathbf{U}_{n+1}^{-n-1} \mathbf{M} \rightarrow \mathbf{U}_{n+1}[1]^{-n-2} \mathbf{M} \rightarrow 0
$$

we have

$$
\left(\mathbf{U}_{n+1}[1]^{-n-2} \mathbf{M}\right)_{\mathfrak{p}}=0 .
$$

$(\Leftarrow) \quad$ By the assumption and the short exact sequences $(*)(* *)$, we have

$$
\left(\mathbf{U}_{n}[1]^{-n-1} \mathbf{M}\right)_{\mathfrak{p}} \cong\left(\operatorname{Im} e^{n}\right)_{\mathfrak{p}} \cong\left(\mathbf{U}_{n+1}^{-n-1} \mathbf{M}\right)_{\mathfrak{p}}
$$

(2) The first equivalence follows immediately from the above short exact sequence $(* *)$. For the second half, this follows from

$$
\operatorname{Supp}\left(\mathbf{U}_{n+1}[1]^{-n-2} \mathbf{M}\right)=\operatorname{Supp}\left(\mathbf{H}_{U}^{n+1}(\mathbf{M})\right) \cup \operatorname{Supp}\left(\mathbf{U}_{n+2}^{-n-2} \mathbf{M}\right)
$$

induced by the short exact sequence $(*)$ with $n+1$ instead of $n$ and Lemma (2.8) (3). 
(3) This follows similarly from the short exact sequence $(*)$ with $n$ replaced by $n+1$.

(4) Suppose that (i) holds. By the hypothesis and Lemma (1.6)(2) we have $\left(\mathbf{U}_{n+1}[1]^{-n-2} \mathbf{M}\right)_{\mathfrak{p}}=0$. On the other hand, from the assumption and Lemma (2.7), we have $\left(H_{U}^{n}(\mathbf{M})\right)_{\mathfrak{p}}=0$.

The other assertions are obvious.

(5) This follows from the hypothesis, Lemma (2.1) and (4).

(6) This follows easily from (2), since $\left(H_{U}^{n+1}(\mathbf{M})\right)_{\mathfrak{p}}=0$.

Example (2.11). (1) In Proposition (2.10), (ii) dose not imply (i). Let $\mathbf{R}=$ $k[[X, Y]]$. Let $\mathbf{M}$ be the quotient field of $\mathbf{R}$. Let $\mathbf{U}_{1}=\mathbf{R} \backslash(X)$ and $\mathfrak{p}=(X, Y)$. Then $\left(\mathbf{U}_{1}^{-1} \mathbf{M}\right)_{\mathfrak{p}}=\mathbf{M}=\left(\mathbf{U}_{0}[1]^{-1} \mathbf{M}\right)_{\mathfrak{p}}=\left(\operatorname{Im} e^{0}\right)_{\mathfrak{p}}$ but $\mathbf{U}_{1} \cap \mathfrak{p} \neq \varnothing$.

(2) $\left(\left(\right.\right.$ iii) $\Rightarrow$ (ii)) is not the case. See Example (2.3) and note that $\left(\mathbf{U}_{f}\right)_{d+1}[1]^{-d-2} \mathbf{M}$ $=0$. When $\mathfrak{p} \in \operatorname{Supp}\left(\mathbf{U}_{n+1}^{-n-1} \mathbf{M}\right)$, we don't know whether this holds or not.

(3) If $\mathfrak{p} \in \operatorname{Supp}\left(\mathbf{U}_{n+2}^{-n-2} \mathbf{M}\right)$, then ((iv) $\Rightarrow$ (iii)) does not hold. Let $(\mathbf{R}, \mathfrak{m})$ be a Buchsbaum ring of dimension $d \geq 3$ such that $\mathbf{H}_{\mathrm{m}}^{1}(\mathbf{R}) \neq 0$ and $\mathbf{H}_{\mathrm{m}}^{n}(\mathbf{R})=0$ for $n \neq 1, d$. Let $U_{f}=\left(\left(\mathbf{U}_{f}\right)_{i}\right)_{i \geq 1}$ be the chain of triangular subsets on $\mathbf{R}$ in the following Proposition (2.15) (when $\mathbf{M}=\mathbf{R}$ ). Then by Proposition (2.15) we have $\operatorname{Ker} f^{1} / \operatorname{Im} f^{0}=\mathbf{H}_{\mathfrak{m}}^{1}(\mathbf{R}) \neq 0$ and $\operatorname{Ker} f^{n} / \operatorname{Im} f^{n-1}=\mathbf{H}_{\mathfrak{m}}^{n}(\mathbf{R})=0$ for $n \neq 1, d$. Hence by the short exact sequence $(*)$ we have

$$
\left(\mathbf{U}_{f}\right)_{n+1}[1]^{-n-2} \mathbf{R} \cong \operatorname{Im} f^{n+1}
$$

for $n \neq 0, d-1$. Let $\mathfrak{p} \in \operatorname{Spec}(\mathbf{R})$ such that ht $\mathfrak{p}=n+1$ for $n=1, \ldots, d-2$. Then $\left(\operatorname{Im} f^{n+1}\right)_{\mathfrak{p}} \neq 0$ since $\operatorname{Supp}\left(\operatorname{Im} f^{n+1}\right)=\operatorname{Supp}\left(\left(\mathbf{U}_{f}\right)_{n+2}^{-n-2} \mathbf{R}\right)=\mathbf{F}_{\mathbf{R}(n+1)}$ by Lemma (2.8)(3) and ([C], 2.15). Therefore $\mathfrak{p} \in \operatorname{Supp}\left(\left(\mathbf{U}_{f}\right)_{n+1}[1]^{-n-2} \mathbf{R}\right)$.

(4) In general, the converse of Proposition (2.10)(5) is not true. Let $\mathbf{R}=$ $k[[X, Y, Z]] /(X) \cap(Y, Z)=k[[x, y, z]]$. Then Ass $(\mathbf{R})=\{(x),(y, z)\}$. Put $\mathfrak{p}=(x, y, z)$ and $\mathbf{U}_{1}=\mathbf{R} \backslash \mathfrak{p}$. Hence $\operatorname{Ass}\left(\mathbf{R}_{\mathfrak{p}}\right)=\{(x),(y, z)\}$. Let $\mathfrak{q}=(x, y)$. Then $\left(\mathbf{U}_{1}^{-1} \mathbf{R}\right)_{\mathfrak{q}}=\left(\mathbf{R}_{\mathrm{p}}\right)_{\mathrm{q}}=\mathbf{R}_{\mathrm{q}}=\left(\mathbf{U}_{0}[1]^{-1} \mathbf{R}\right)_{\mathrm{q}}=\left(\operatorname{Im} e^{0}\right)_{\mathrm{q}} \neq 0$ and $\mathbf{U}_{1} \cap q=\emptyset$. But $q \notin \operatorname{Ass}\left(\mathbf{R}_{p}\right)$.

Corollary (2.12). Let $\mathbf{R}$ be a Noetherian ring and $\mathbf{M}$ an $\mathbf{R}$-module. Then we have the following.

(1) $\operatorname{Ass}\left(\mathbf{U}_{n+1}^{-n-1} \mathbf{M}\right) \subset \operatorname{Ass}\left(\mathbf{U}_{n}[1]^{-n-1} \mathbf{M}\right)$.

(2) $\operatorname{Ass}\left(\mathbf{U}_{n}[1]^{-n-1} \mathbf{M}\right)=\operatorname{Ass}\left(H_{U}^{n}(\mathbf{M})\right) \cup \operatorname{Ass}\left(\mathbf{U}_{n+1}^{-n-1} \mathbf{M}\right)$.

Proof. (1) Let $\mathfrak{p} \in \operatorname{Ass}_{\mathbf{R}}\left(\mathbf{U}_{n+1}^{-n-1} \mathbf{M}\right)$. Then $\mathfrak{p R}_{\mathfrak{p}} \in \operatorname{Ass}_{\mathbf{R}_{\mathfrak{p}}}\left(\mathbf{U}_{n+1}^{-n-1} \mathbf{M}\right)_{\mathfrak{p}}$ by ([M], p. 38 Corollary). Hence $\mathfrak{p} \mathbf{R}_{\mathfrak{p}} \in \operatorname{Ass}_{\mathbf{R}_{\mathfrak{p}}}\left(\mathbf{U}_{n}[1]^{-n-1} \mathbf{M}\right)_{\mathfrak{p}}$ by Proposition (2.10)(5). 
Therefore $\mathfrak{p} \in \operatorname{Ass}_{\mathbf{R}}\left(\mathbf{U}_{n}[1]^{-n-1} \mathbf{M}\right)$ again by ([M], p. 38 Corollary).

(2) This follows from (1) and Lemma (2.8)(5).

Proposition (2.13). Let $\mathbf{R}$ be a ring and $\mathbf{M}$ an $\mathbf{R}$-module. Fix a non-negative integer $t$. Then in $\mathbf{C}(\mathcal{U}, \mathbf{M})$, the following four conditions are equivalent.

(1) $H_{U}^{n}(\mathbf{M})=0$ for all $n=0, \ldots, t$.

(2) $\mathbf{U}_{n}[1]^{-n-1} \mathbf{M} \cong \operatorname{Im} e^{n}$ for all $n=0, \ldots, t$.

(3) For all $n=0, \ldots$, t, for each $\frac{m}{\left(a_{1}, \ldots, a_{n+1}\right)} \in \mathbf{U}_{n+1}^{-n-1} \mathbf{M}$,

$\left(0: \frac{m}{\left(a_{1}, \ldots, a_{n+1}\right)}\right)=\left(0: \frac{m}{\left(a_{1}, \ldots, a_{n}, 1\right)}\right)$ where $\frac{m}{\left(a_{1}, \ldots, a_{n}, 1\right)} \in \mathbf{U}_{n}[1]^{-n-1} \mathbf{M}$.

(4) For all $n=0, \ldots, t$, each element of $\mathbf{U}_{n+1}$ forms a poor $\mathbf{M}$-sequence.

In particular, let $\mathbf{R}$ be a Noetherian local ring and let $\mathbf{M}$ be a finitely generated $\mathbf{R}$-module of dimension $d$. Assume that the above conditions hold for $t=d-1$ and $\mathbf{U}_{d}[1]^{-d-1} \mathbf{M} \neq 0$. Then $\mathbf{M}$ is a Cohen-Macaulay module.

Proof. (1) $\Leftrightarrow(2)$ From the short exact sequence $(*)$ this is clear.

$(2) \Rightarrow(3)$ By Lemma (1.6)(3) this is obvious.

$(3) \Rightarrow(4)$ We proceed by induction on $n$. In the case $n=0$, assume that $a_{1} m=0$ for some $0 \neq m \in \mathbf{M}$ and $\left(a_{1}\right) \in \mathbf{U}_{1}$. Then we have $a_{1} \in(0: m)=$ $\left(0: \frac{m}{\left(b_{1}\right)}\right)$ for some $\frac{m}{\left(b_{1}\right)} \in \mathbf{U}_{1}^{-1} \mathbf{M}$ by the hypothesis. This contradicts Lemma (2.1).

Now suppose that each element of $\mathbf{U}_{n}$ is a poor $\mathbf{M}$-sequence. Assume that $a_{n+1} m \in\left(a_{1}, \ldots, a_{n}\right) \mathbf{M}$ for some $\left(a_{1}, \ldots, a_{n+1}\right) \in \mathbf{U}_{n+1}$ and $m \in \mathbf{M}$. Then by Lemma (1.6)(2) we have $\frac{a_{n+1} m}{\left(a_{1}, \ldots, a_{n+1}\right)}=0$. That is, by ([SZ3], 2.1), we have

$$
\frac{m}{\left(a_{1}, \ldots, a_{n+1}\right)}=0 \text { in } \mathbf{U}_{n+1}^{-n-1} \mathbf{M} \text {. }
$$

Hence by the hypothesis we have

$$
\frac{m}{\left(a_{1}, \ldots, a_{n}, 1\right)}=0 \text { in } \mathbf{U}_{n}[1]^{-n-1} \mathbf{M}
$$

Then, by the definition of module of generalized fractions, there are $\left(b_{1}, \ldots, b_{n}, 1\right)$ $\in \mathbf{U}_{n}[1]$ and $\mathbf{H} \in \mathbf{D}_{n+1}(\mathbf{R})$ such that $\mathbf{H}\left[a_{1} \ldots a_{n} 1\right]^{T}=\left[b_{1} \ldots b_{n} 1\right]^{T}$ and $|\mathbf{H}| m \in\left(b_{1}, \ldots, b_{n}\right) \mathbf{M}$. 
On the other hand, since $h_{n+1, n+1}=1-\left(h_{n+1,1} a_{1}+\cdots+h_{n+1, n} a_{n}\right)$, by ([SZ1], 2.2) we have

$$
h_{11} \cdots h_{n n} m \in\left(b_{1}, \ldots, b_{n}\right) \mathbf{M}
$$

Note that by the inductive hypothesis $b_{1}, \ldots, b_{n}$ is a poor $\mathbf{M}$-sequence and $\mathbf{H}^{\prime}\left[a_{1}\right.$ $\left.\ldots a_{n}\right]^{T}=\left[b_{1} \ldots b_{n}\right]^{T}$ where $\mathbf{H}^{\prime}$ is the top left $n \times n$ submatrix of $\mathbf{H}$. Hence by ([O], 3.2) we get

$$
m \in\left(a_{1}, \ldots, a_{n}\right) \mathbf{M}
$$

$(4) \Rightarrow(1)$ Let $\frac{m}{\left(a_{1}, \ldots, a_{n}\right)} \in \operatorname{Ker} e^{n}$ with $\frac{m}{\left(a_{0}\right)}=m$. Then $\frac{m}{\left(a_{1}, \ldots, a_{n}, 1\right)}=$ 0 in $\mathbf{U}_{n+1}^{-n-1} \mathbf{M}$. Hence by Lemma (1.6)(2), we have

$$
m \in\left(a_{1}, \ldots, a_{n}\right) \mathbf{M}
$$

Therefore we have $\frac{m}{\left(a_{1}, \ldots, a_{n}\right)} \in \operatorname{Im} e^{n-1}$.

For the last assertion, since $\mathbf{U}_{d}[1]^{-d-1} \mathbf{M} \neq 0$, there is $\left(a_{1}, \ldots, a_{d}\right) \in \mathbf{U}_{d}$ such that $a_{1}, \ldots, a_{d}$ is an $\mathbf{M}$-sequence.

Remark (2.14). In Proposition (2.13), if $\mathbf{R}$ is Noetherian, then we can change the condition (3) for $\operatorname{Ass}\left(\mathbf{U}_{n+1}^{-n-1} \mathbf{M}\right)=\operatorname{Ass}\left(\mathbf{U}_{n}[1]^{-n-1} \mathbf{M}\right)$ for all $n=0, \ldots, t$.

Let $(\mathbf{R}, \mathfrak{m})$ be a Noetherian local ring and let $\mathbf{M}$ be a finitely generated $\mathbf{R}$-module of dimension $d$. Let $U_{f}=\left(\left(\mathbf{U}_{f}\right)_{i}\right)_{i \geq 1}$ be the chain of the expansions of triangular subsets (Example (1.3)(5)) on $\mathbf{R}$. Then we have the following complex

$$
0 \rightarrow \mathbf{M} \stackrel{f^{0}}{\rightarrow}\left(\mathbf{U}_{f}\right)_{1}^{-1} \mathbf{M} \stackrel{f^{1}}{\rightarrow}\left(\mathbf{U}_{f}\right)_{2}^{-2} \mathbf{M} \rightarrow \cdots \rightarrow\left(\mathbf{U}_{f}\right)_{d-1}^{-d+1} \mathbf{M} \stackrel{f^{d-1}}{\rightarrow}\left(\mathbf{U}_{f}\right)_{d}^{-d} \mathbf{M} \stackrel{f^{d}}{\rightarrow} 0
$$

since $\left(\mathbf{U}_{f}\right)_{d+i}^{-d-i} \mathbf{M}=0$ for all $i \geq 1$ by $([C], 2.3)$.

Proposition (2.15). Let $\mathbf{R}, \mathbf{M}$ and $\mathbf{U}_{f}$ be as above. Then the following four conditions are equivalent.

(1) $\mathbf{M}$ is an f-module (see [SZ4], 1.8 (ii)).

(2) $\operatorname{Ker} f^{n} / \operatorname{Im} f^{n-1} \cong \mathbf{H}_{\mathrm{m}}^{n}(\mathbf{M})$ for all $n=0, \ldots, d$.

(3) $\operatorname{Ass}\left(\left(\mathbf{U}_{f}\right)_{n}[1]^{-n-1} \mathbf{M}\right) \subset\{\mathrm{m}\} \cup \operatorname{Ass}\left(\left(\mathbf{U}_{f}\right)_{n+1}^{-n-1} \mathbf{M}\right)$ for all $n=0, \ldots, d$.

(4) $\operatorname{Supp}\left(\operatorname{Ker} f^{n} / \operatorname{Im} f^{n-1}\right) \subset\{\mathrm{m}\}$ for all $n=0, \ldots, d$.

In particular, if $\mathbf{M}$ is a Cohen-Macauly module, then

$$
\left\{\begin{array}{l}
\operatorname{Ass}\left(\left(\mathbf{U}_{f}\right)_{n}[1]^{-n-1} \mathbf{M}\right)=\operatorname{Ass}\left(\left(\mathbf{U}_{f}\right)_{n+1}^{-n-1} \mathbf{M}\right)=\mathbf{F}_{\mathrm{M} n} \quad \text { for all } n<d, \\
\operatorname{Ass}\left(\left(\mathbf{U}_{f}\right)_{d}[1]^{-d-1} \mathbf{M}\right)=\{\mathbf{m}\}
\end{array}\right.
$$


Proof. (1) $\Rightarrow(2)$ In the case $n=0, \ldots, d-1$, this follows from ([SZ4], 2.4), since $\left(\mathbf{U}_{f}\right)_{n}=\left(\mathbf{U}_{s}\right)_{n}$. In the case $n=d$, we have

$$
\operatorname{Ker} f^{d} / \operatorname{Im} f^{d-1} \cong \mathbf{U}_{d}^{-d} \mathbf{M} / \operatorname{Im} f^{d-1} \cong \mathbf{U}_{d}[1]^{-d-1} \mathbf{M} \cong\left(\mathbf{U}_{s}\right)_{d+1}^{-d-1} \mathbf{M} \cong \mathbf{H}_{\mathrm{m}}^{d} \mathbf{M}
$$

by Lemma (1.9) and Lemma (1.7).

$(2) \Rightarrow(3) \Leftrightarrow(4)$ These follow from Corollary (2.12)(2) and Lemma (2.8)(4).

$(4) \Rightarrow(1)$ This follows from ([SZ4], 2.3).

The last assertion follows from (2), Corollary (2.12)(2) and ([C], 2.15).

\section{Modules of generalized fractions and complexes of Cousin type}

In this section, suppose that $\mathbf{R}$ is a Noetherian ring.

Theorem (3.1). Let $\mathbf{R}$ be a Noetherian ring and $\mathbf{M}$ an $\mathbf{R}$-module. Let $\boldsymbol{U}=$ $\left(\mathbf{U}_{i}\right)_{i \geq 1}$ be a chain of triangular subsets on $\mathbf{R}$. Let $\mathscr{F}=\left(\mathbf{F}_{i}\right)_{i \geq 0}$ be a filtration of $\operatorname{Spec}(\mathbf{R})$ which admits $\mathbf{M}$. Then

the complex $\mathbf{C}(\mathcal{U}, \mathbf{M})$ is of Cousin type for $\mathbf{M}$ with respect to $\mathscr{F}$

$$
\begin{aligned}
\operatorname{Ass}\left(\mathbf{U}_{n}[1]^{-n-1} \mathbf{M}\right) & \cap \partial \mathbf{F}_{n}=\operatorname{Ass}\left(\mathbf{U}_{n+1}^{-n-1} \mathbf{M}\right) \text { for all } n \geq 0 \text { and } \\
\mathbf{U}_{n+1}^{-n-1} \mathbf{M} & \cong \underset{\mathfrak{p} \in \partial \mathbf{F}_{n}}{\bigoplus}\left(\mathbf{U}_{n+1}^{-n-1} \mathbf{M}\right)_{p} \text { for all } n \geq 0 .
\end{aligned}
$$

Proof. ( $\uparrow$ ) We must verify the properties (i)-(iii) of the definition of Cousin type (see (1.4)).

(i) and (ii) By Remark (2.5) these always hold for arbitrary complexes $\mathbf{C}(\mathcal{U}, \mathbf{M})$.

(iii) We must show that $\operatorname{Supp}\left(H_{U}^{n}(\mathbf{M})\right) \subset \mathbf{F}_{n+1}$. Note that $\operatorname{Ass}\left(\mathbf{U}_{n+1}^{-n-1} \mathbf{M}\right)=$ $\operatorname{Ass}\left(\underset{p \in \partial \mathbf{F}_{n}}{\bigoplus}\left(\mathbf{U}_{n+1}^{-n-1} \mathbf{M}\right)_{\mathfrak{p}}\right) \subset \partial \mathbf{F}_{n}$ by Lemma (2.4). By Lemma (2.8)(5) and Lemma (2.4), we have $\operatorname{Supp}\left(H_{U}^{n}(\mathbf{M})\right) \subset \operatorname{Supp}\left(\mathbf{U}_{n}[1]^{-n-1} \mathbf{M}\right) \subset \mathbf{F}_{n}$. But it follows from the hypothesis and Lemma (2.8)(4)(6) that $\partial \mathbf{F}_{n} \cap \operatorname{Supp}\left(H_{U}^{n}(\mathbf{M})\right)=\varnothing$.

$(\downarrow)$ It is enough to show that the first condition of Theorem holds. By the third and the fourth conditions of the definition of Cousin type, we have $\partial \mathbf{F}_{n} \cap$ $\operatorname{Supp}\left(H_{U}^{n}(\mathbf{M})\right)=\varnothing$ and $\operatorname{Ass}\left(\mathbf{U}_{n+1}^{-n-1} \mathbf{M}\right) \subset \partial \mathbf{F}_{n}$. Hence Lemma (2.8)(6) completes the proof of Theorem. 
COROLlary (3.2). With the same notation and assumption as in Theorem (3.1), we have the following.

(1) Suppose that $\partial \mathbf{F}_{\mathrm{M} n} \cap \partial \mathbf{F}_{n}=\operatorname{Ass}\left(\mathbf{U}_{n+1}^{-n-1} \mathbf{M}\right)$ for all $n \geq 0$ and

$$
\mathbf{U}_{n+1}^{-n-1} \mathbf{M} \cong \bigoplus_{\mathfrak{p} \in \partial \mathbf{F}_{n}}\left(\mathbf{U}_{n+1}^{-n-1} \mathbf{M}\right)_{\mathfrak{p}} \text { for all } n \geq 0 .
$$

Then the complex $\mathbf{C}(\mathcal{U}, \mathbf{M})$ is of Cousin type for $\mathbf{M}$ with respect to $\mathscr{F}$.

(2) In particular, assume that $\partial \mathbf{F}_{\mathrm{M} n} \cap \partial \mathbf{F}_{n} \subset \operatorname{Supp}\left(\mathbf{U}_{n}[1]^{-n-1} \mathbf{M}\right)$ for all $n \geq 0$.

Then the converse of (1) is true.

Proof. (1) This follows from Theorem (3.1), since $\operatorname{Ass}\left(\mathbf{U}_{n+1}^{-n-1} \mathbf{M}\right) \subset$ $\operatorname{Ass}\left(\mathbf{U}_{n}[1]^{-n-1} \mathbf{M}\right) \cap \partial \mathbf{F}_{n} \subset \partial \mathbf{F}_{\mathrm{M} n} \cap \partial \mathbf{F}_{n}$ by Corollary (2.12)(1) and Lemma (2.6)(2).

(2) It is sufficient to show that $\partial \mathbf{F}_{\mathrm{M} n} \cap \partial \mathbf{F}_{n}=\operatorname{Ass}\left(\mathbf{U}_{n+1}^{-n-1} \mathbf{M}\right)$, since the second isomorphisms hold by the definition of Cousin type.

(د) Since Ass $\left(\mathbf{U}_{n+1}^{-n-1} \mathbf{M}\right) \subset \partial \mathbf{F}_{n}$, it follows from Lemma (2.6)(2) that $\operatorname{Ass}\left(\mathbf{U}_{n+1}^{-n-1} \mathbf{M}\right) \subset \partial \mathbf{F}_{\mathrm{M} n} \cap \partial \mathbf{F}_{n}$.

(c) We proceed by induction on $n$. In the case $n=0$, let $\mathfrak{p} \in \partial \mathbf{F}_{\mathrm{M} 0} \cap \partial \mathbf{F}_{0}$. Consider the following complex

$$
0 \rightarrow \mathbf{M} \stackrel{e^{0}}{\rightarrow} \mathbf{U}_{1}^{-1} \mathbf{M} \stackrel{e^{1}}{\rightarrow} \mathbf{U}_{2}^{-2} \mathbf{M} \rightarrow \cdots
$$

Then by the definition of Cousin type, we have the following exact sequence

$$
0 \rightarrow \mathbf{M}_{\mathfrak{p}} \cong\left(\mathbf{U}_{1}^{-1} \mathbf{M}\right)_{\mathfrak{p}} \rightarrow 0 \text {. }
$$

Since $\mathfrak{p} \in \operatorname{Ass}(\mathbf{M})$, we have $\mathfrak{p} \in \operatorname{Ass}\left(\mathbf{U}_{1}^{-1} \mathbf{M}\right)$ by ([M], p. 38 Corollary).

Suppose that $n \geq 1$. Let $\mathfrak{p} \in \partial \mathbf{F}_{\mathrm{M} n} \cap \partial \mathbf{F}_{n}$. Consider the following complex

$$
\cdots \rightarrow \mathbf{U}_{n-1}^{-n+1} \stackrel{e^{n-1}}{\rightarrow} \mathbf{U}_{n}^{-n} \mathbf{M} \stackrel{e^{n}}{\rightarrow} \mathbf{U}_{n+1}^{-n-1} \mathbf{M} \rightarrow \cdots
$$

It follows from the definition of Cousin type that we have the following exact sequence

$$
0 \rightarrow\left(\operatorname{Im} e^{n-1}\right)_{\mathfrak{p}} \rightarrow\left(\mathbf{U}_{n}^{-n} \mathbf{M}\right)_{\mathfrak{p}} \rightarrow\left(\mathbf{U}_{n+1}^{-n-1} \mathbf{M}\right)_{\mathfrak{p}} \rightarrow 0
$$

since $\left(\operatorname{Ker} e^{n}\right)_{p} \cong\left(\operatorname{Im} e^{n-1}\right)_{p}$. Hence by the inductive hypothesis and Lemma (2.6) (3), we have $\left(\mathbf{U}_{n}^{-n} \mathbf{M}\right)_{\mathfrak{p}} \neq 0$. On the other hand, by Proposition (2.10)(2) and the assumption $\partial \mathbf{F}_{\mathrm{M} n} \cap \partial \mathbf{F}_{n} \subset \operatorname{Supp}\left(\mathbf{U}_{n}[1]^{-n-1} \mathbf{M}\right)$, we get

$$
\left(\operatorname{Im} e^{n-1}\right)_{\mathfrak{p}} \not\left(\mathbf{U}_{n}^{-n} \mathbf{M}\right)_{\mathfrak{p}} .
$$


That is $\left(\mathbf{U}_{n+1}^{-n-1} \mathbf{M}\right)_{\mathfrak{p}} \neq 0$. Hence we conclude that $\mathfrak{p} \in \operatorname{Ass}\left(\mathbf{U}_{n+1}^{-n-1} \mathbf{M}\right)$ by Lemma (2.4).

Remark (3.3). Using Lemma (2.6)(2), Lemma (2.8)(6), the third and the fourth conditions of the definition of Cousin type, we have another proof of Corollary (3.2)(2) as follows:

$$
\begin{aligned}
\partial \mathbf{F}_{\mathrm{M} n} \cap \partial \mathbf{F}_{n} & =\partial \mathbf{F}_{n} \cap \operatorname{Supp}\left(\mathbf{U}_{n}[1]^{-n-1} \mathbf{M}\right)=\partial \mathbf{F}_{n} \cap \operatorname{Ass}\left(\mathbf{U}_{n}[1]^{-n-1} \mathbf{M}\right) \\
& =\left(\partial \mathbf{F}_{n} \cap\left(H_{U}^{n}(\mathbf{M})\right)\right) \cup\left(\partial \mathbf{F}_{n} \cap \operatorname{Ass}\left(\mathbf{U}_{n+1}^{-n-1} \mathbf{M}\right)\right) \\
& =\partial \mathbf{F}_{n} \cap \operatorname{Ass}\left(\mathbf{U}_{n+1}^{-n-1} \mathbf{M}\right)=\operatorname{Ass}\left(\mathbf{U}_{n+1}^{-n-1} \mathbf{M}\right)
\end{aligned}
$$

Remark (3.4). If $\mathbf{M}$ is a finitely generated $\mathbf{R}$-module and a complex $\mathbf{C}(\mathcal{U}, \mathbf{M})$ is of Cousin type for $\mathbf{M}$ with respect to $\mathscr{F}_{\mathrm{M}}$, then $\operatorname{Ass}\left(\mathbf{U}_{n+1}^{-n-1} \mathbf{M}\right)=\{\mathfrak{p} \in$ $\left.\operatorname{Supp}(\mathbf{M}): \mathrm{ht}_{\mathbf{M}} \mathfrak{p}=n\right\}$ by ([RSZ], 3.3), ([C], 2.11) and the following Corollary (3.5) (1).

Corollary (3.5). Let $\mathbf{M}$ be a finitely generated $\mathbf{R}$-module of dimension $d$. Let $\mathscr{F}=\left(\mathbf{F}_{i}\right)_{i \geq 0}$ be a filtration of $\operatorname{Spec}(\mathbf{R})$ which admits $\mathbf{M}$. Let $\mathscr{F}_{\mathbf{M}}=\left(\mathbf{F}_{\mathbf{M} i}\right)_{i \geq 0}$ be the M-height filtration.

(1) (cf. [SY], 3.9) $\mathbf{C}\left(\mathcal{U}_{h}, \mathbf{M}\right)$ is of Cousin type for $\mathbf{M} w . r$. t. $\mathscr{F}_{\mathbf{M}}$, where $\mathscr{U}_{h}=$ $\left(\left(\mathbf{U}_{h}\right)_{i}\right)_{i \geq 0}$.

(2) ([RSZ], 3.4) $\mathbf{C}\left(\mathcal{U}_{\bar{h}}, \mathbf{M}\right)$ is of Cousin type for $\mathbf{M}$ w. r. t. $\mathscr{F}$, where $\mathcal{U}_{\bar{h}}=\left(\left(\mathbf{U}_{\bar{h}}\right)_{i}\right)_{i \geq 0}$.

(3) ([GO], 3.6) Let $\mathcal{U}=\left(\mathbf{U}_{i}\right)_{i \geq 0}$ be a chain of saturated triangular subsets on $\mathbf{R}$. Put $\mathbf{G}_{0}=\operatorname{Supp}(\mathbf{M})$ and for $i \in \mathbf{N}$, define $\mathbf{G}_{i}=\{\mathfrak{p} \in \operatorname{Supp}(\mathbf{M})$ : there exists $\left(a_{1}, \ldots, a_{i}\right) \in \mathbf{U}_{i}$ with $\left.\left(a_{1}, \ldots, a_{i}\right) \mathbf{R} \subset \mathfrak{p}\right\}$. Assume that $\mathscr{G}=\left(\mathbf{G}_{i}\right)_{i \geq 0}$, induced by $\mathcal{U}$ and $\mathbf{M}$, is a filtration of $\operatorname{Spec}(\mathbf{R})$ which admits $\mathbf{M}$. Then

$\mathbf{C}(\mathcal{U}, \mathbf{M})$ is of Cousin type for $\mathbf{M}$ w.r.t. $\mathscr{G}$.

(4) If $\operatorname{dim} \mathbf{M}=\mathrm{ht}_{\mathbf{M}} \mathfrak{q}+\operatorname{dim} \mathbf{M} / \mathfrak{q} \mathbf{M}$ for all $\mathfrak{q} \in \operatorname{Supp}(\mathbf{M})$, then

$\mathbf{C}\left(\mathcal{U}_{s}, \mathbf{M}\right)$ is of Cousin type for $\mathbf{M}$ w.r.t. $\mathscr{F}_{\mathrm{M}}$, where $\mathcal{U}_{s}=\left(\left(\mathbf{U}_{s}\right)_{i}\right)_{i \geq 0}$.

(5) Let $\mathcal{U}_{r}=\left(\left(\mathbf{U}_{r}\right)_{i}\right)_{i \geq 0}$. Then we have the following equivalent conditions.

$\mathbf{M}$ is a Cohen-Macaulay module

$\Leftrightarrow \mathbf{C}\left(\mathcal{U}_{r}, \mathbf{M}\right)$ is of Cousin type for $\mathbf{M}$ w.r.t. $\mathscr{F}_{\mathbf{M}}$

$$
\Leftrightarrow\left(\mathbf{U}_{r}\right)_{n+1}^{-n-1} \mathbf{M} \cong \underset{\mathrm{ht}_{\mathbf{M}_{\mathfrak{p}}=n}}{\bigoplus}\left(\left(\mathbf{U}_{r}\right)_{n+1}^{-n-1} \mathbf{M}\right)_{\mathfrak{p}} \text { for all } n \geq 0 \text {. }
$$

(6) Let $\mathbf{R}$ be a Noetherian local ring. Then

$\mathbf{M}$ is a Gorenstein module

$\Leftrightarrow\left\{\begin{array}{l}\mathbf{C}\left(\mathcal{U}_{r}, \mathbf{M}\right) \text { is of Cousin type for } \mathbf{M} \text { w.r.t. } \mathscr{F}_{\mathbf{M}} \text { and } \\ \left(\mathbf{U}_{r}\right)_{d+1}^{-d-1} \mathbf{M} \text { is an injective } \mathbf{R} \text {-module }\end{array}\right.$ 


$$
\Leftrightarrow\left\{\begin{array}{l}
\left(\mathbf{U}_{r}\right)_{n+1}^{-n-1} \mathbf{M} \cong \underset{\mathrm{ht}_{\mathbf{M}} \mathfrak{p}=n}{\bigoplus}\left(\left(\mathbf{U}_{r}\right)_{n+1}^{-n-1} \mathbf{M}\right)_{\mathfrak{p}} \text { for all } n \geq 0 \text {, and } \\
\left(\mathbf{U}_{r}\right)_{d+1}^{-d-1} \mathbf{M} \text { is an injective } \mathbf{R} \text {-module. }
\end{array}\right.
$$

Proof. (1) This follows from ([C], 2.11 and 3.3(2)) and Corollary (3.2).

(2) By ([RSZ], 2.6 or [C], 3.3(1)), we have for all $n \in \mathbf{N} \cup\{0\}$

$$
\left(\mathbf{U}_{\bar{h}}\right)_{n+1}^{-n-1} \mathbf{M} \cong \bigoplus_{\mathfrak{p} \in \partial \mathbf{F}_{n}}\left(\left(\mathbf{U}_{\bar{h}}\right)_{n+1}^{-n-1} \mathbf{M}\right)_{\mathfrak{p}}
$$

Hence by Lemma (2.4) we get

$$
\begin{aligned}
\operatorname{Ass}\left(\left(\mathbf{U}_{\bar{h}}\right)_{n+1}^{-n-1} \mathbf{M}\right) & =\operatorname{Ass}\left(\bigoplus_{\mathfrak{p} \in \partial \mathbf{F}_{n}}\left(\left(\mathbf{U}_{\bar{h}}\right)_{n+1}^{-n-1} \mathbf{M}\right)_{\mathfrak{p}}\right) \\
& =\underset{p \in \partial \mathbf{F}_{n}}{\cup} \operatorname{Ass}\left(\left(\left(\mathbf{U}_{\bar{h}}\right)_{n+1}^{-n-1} \mathbf{M}\right)_{\mathfrak{p}}\right) \subset \partial \mathbf{F}_{n} .
\end{aligned}
$$

By Lemma (2.7) and the definition of $\left(\mathbf{U}_{\bar{h}}\right)_{n+1}$, we have, for all $\mathfrak{p} \in \partial \mathbf{F}_{n} \cap$ $\operatorname{Supp}(\mathbf{M})$,

$$
\left(H_{U}^{n}(\mathbf{M})\right)_{\mathfrak{p}}=0 .
$$

Therefore we have $\partial \mathbf{F}_{n} \cap \operatorname{Ass}\left(H_{U}^{n}(\mathbf{M})\right)=\emptyset$, since $\operatorname{Ass}\left(H_{U}^{n}(\mathbf{M})\right) \subset \operatorname{Supp}(\mathbf{M})$. Hence we obtain

$$
\partial \mathbf{F}_{n} \cap \operatorname{Ass}\left(\left(\mathbf{U}_{\bar{h}}\right)_{n}[1]^{-n-1} \mathbf{M}\right)=\partial \mathbf{F}_{n} \cap \operatorname{Ass}\left(\left(\mathbf{U}_{\bar{h}}\right)_{n+1}^{-n-1} \mathbf{M}\right)=\operatorname{Ass}\left(\left(\mathbf{U}_{\bar{h}}\right)_{n+1}^{-n-1} \mathbf{M}\right),
$$

by Lemma (2.8) (6). Then Theorem (3.1) completes the proof.

(3) By ([GO], 3.6), we have for all $n \in \mathbf{N} \cup\{0\}$

$$
\mathbf{U}_{n+1}^{-n-1} \mathbf{M} \cong \underset{\mathfrak{p} \in \partial \mathbf{G}_{n}}{\bigoplus}\left(\mathbf{U}_{n+1}^{-n-1} \mathbf{M}\right)_{\mathfrak{p}}
$$

Hence we get Ass $\left(\mathbf{U}_{n+1}^{-n-1} \mathbf{M}\right) \subset \partial \mathbf{G}_{n}$.

Next for all $\mathfrak{p} \in \partial \mathbf{G}_{n}$ we have

$$
\left(H_{U}^{n}(\mathbf{M})\right)_{p}=0 .
$$

In fact, if $\left(H_{U}^{n}(\mathbf{M})\right)_{\mathfrak{p}} \neq 0$, then there is $x \in H_{U}^{n}(\mathbf{M})$ such that $(0: x) \subset \mathfrak{p}$. But by Lemma (2.7), we have $\left(a_{1}, \ldots, a_{n+1}\right) \mathbf{R} \subset(0: x) \subset \mathfrak{p}$ for some $\left(a_{1}, \ldots, a_{n+1}\right) \in$ $\mathbf{U}_{n+1}$. Hence from the definition of $\mathbf{G}_{n+1}$ we have $\mathfrak{p} \in \mathbf{G}_{n+1}$. This contradicts $\mathfrak{p} \in \partial \mathbf{G}_{n}$.

Therefore we have $\partial \mathbf{G}_{n} \cap \operatorname{Ass}\left(H_{U}^{n}(\mathbf{M})\right)=\emptyset$.

Then by Lemma (2.8)(6) we get

$$
\begin{aligned}
\partial \mathbf{G}_{n} \cap \operatorname{Ass}\left(\mathbf{U}_{n}[1]^{-n-1} \mathbf{M}\right) & =\left(\partial \mathbf{G}_{n} \cap \operatorname{Ass}\left(H_{U}^{n}(\mathbf{M})\right)\right) \cup\left(\partial \mathbf{G}_{n} \cap \operatorname{Ass}\left(\mathbf{U}_{n+1}^{-n-1} \mathbf{M}\right)\right) \\
& =\operatorname{Ass}\left(\mathbf{U}_{n+1}^{-n-1} \mathbf{M}\right) .
\end{aligned}
$$


The result follows from Theorem (3.1).

(4) This follows from ([C], 2.12 and 3.3(3)) and Corollary (3.2).

(5) Since $\mathbf{C}\left(\mathcal{U}_{r}, \mathbf{M}\right)$ is an exact sequence by Proposition (2.13), the first equivalence follows from ([S2], 2.4). From Proposition (2.13)(3) and Theorem (3.1), we have the second equivalence.

(6) This follows from (5) and ([S2], 3.11).

Remark (3.6). Let $(\mathbf{R}, \mathfrak{m})$ be a Noetherian ring and let $\mathbf{M}$ be a finitely generated $f$-module of dimension $d$. Then $\mathbf{C}\left(\mathcal{U}_{s}, \mathbf{M}\right)$ is of Cousin type for $\mathbf{M}$ with respect to $\mathscr{F}_{\mathbf{M}}$ (Corollary (3.5)(4)) but $\mathbf{C}\left(\mathcal{U}_{f}, \mathbf{M}\right)$ is not, even though $\left(\mathbf{U}_{f}\right)_{n+1}^{-n-1} \mathbf{M} \cong$ $\bigoplus_{\mathrm{ht}_{\mathbf{M}}=n}\left(\left(\mathbf{U}_{f}\right)_{n+1}^{-n-1} \mathbf{M}\right)_{\mathfrak{p}}$ for all $n \geq 0$ ([C], 3.3(5)). For, by ([C], 2.15), we have $\operatorname{Ass}\left(\left(\mathbf{U}_{f}\right)_{d+1}^{-d-1} \mathbf{M}\right)=\emptyset$ but $\operatorname{Ass}\left(\left(\mathbf{U}_{f}\right)_{d}[1]^{-d-1} \mathbf{M}\right) \cap \partial \mathbf{F}_{\mathbf{M} d}=\operatorname{Ass}\left(\left(\mathbf{U}_{s}\right)_{d+1}^{-d-1} \mathbf{M}\right) \cap$ $\partial \mathbf{F}_{\mathrm{M} d}=\{\mathrm{m}\}$. Hence we have

$$
\operatorname{Ass}\left(\left(\mathbf{U}_{f}\right)_{d}[1]^{-d-1} \mathbf{M}\right) \cap \partial \mathbf{F}_{\mathrm{M} d} \neq \operatorname{Ass}\left(\left(\mathbf{U}_{f}\right)_{d+1}^{-d-1} \mathbf{M}\right) .
$$

Therefore the result follows from Theorem (3.1).

Example (3.7). Let $\mathbf{R}=k[[x, y, z]]$. Let $\mathbf{U}_{1}=\left\{\left(t x^{\alpha}\right) \in \mathbf{R}^{1}: 0 \neq t \in k\right.$ and $\alpha \in \mathbf{N} \cup\{0\}\}$. Let $\mathbf{U}_{i}=\mathbf{U}_{\imath-1}[1]$ for $i=2,3, \ldots$ Then $\mathcal{U}=\left(\mathbf{U}_{\imath}\right)_{\imath \geq 1}$ is a chain of saturated triangular subsets on $\mathbf{R}$. Put $\mathbf{G}_{0}=\operatorname{Spec}(\mathbf{R}), \mathbf{G}_{1}=\{\mathfrak{p} \in \operatorname{Spec}(\mathbf{R}): x$ $\in \mathfrak{p}\}$ and $\mathbf{G}_{\imath}=\emptyset$ for $i \geq 2$. Then $\mathscr{G}=\left(\mathbf{G}_{i}\right)_{i \geq 0}$ is induced by $\mathcal{U}$ and $\mathbf{M}$ as in (3) of Corollary (3.5), but is not a filtration of $\operatorname{Spec}(\mathbf{R})$. For, $\partial \mathbf{G}_{0}=\mathbf{G}_{0} \backslash \mathbf{G}_{1} \supset\{(y)$, $(y, z)\}$.

Example (3.8). Let $\mathbf{R}=k[[X, Y, Z]] /(X) \cap(Y, Z)=k[[x, y, z]]$. Then $\mathbf{R}$ is not equidimensional and $\{(x),(y, z)\}=\partial \mathbf{F}_{\mathbf{R} 0} \cap \operatorname{Spec}\left(\left(\mathbf{U}_{s}\right)_{0}[1]^{-1} \mathbf{R}\right) \not \subset$ $\operatorname{Ass}\left(\left(\mathbf{U}_{s}\right)_{1}^{-1} \mathbf{R}\right)=\{(x)\}$. Hence $\mathbf{C}\left(U_{s}, \mathbf{R}\right)$ is not of Cousin type for $\mathbf{R}$ w. r. t. $\mathscr{F}_{\mathbf{R}}$. In fact, $k((y, z)) \times k((x)) \cong\left(\mathbf{U}_{h}\right)_{1}^{-1} \mathbf{R} ¥\left(\mathbf{U}_{s}\right)_{1}^{-1} \mathbf{R} \cong k((y, z))$ (cf. Corollary $(3.5)(1)$ (4)).

Example (3.9). Let $\mathbf{R}=k[[x, y]]$. Let $\mathbf{U}_{1}=\left\{\left(x^{\alpha}\right) \in \mathbf{R}^{1}: \alpha \in \mathbf{N} \cup\right.$ $\{0\}\}$ and $\mathbf{U}_{n}=\left\{\left(x^{\alpha}, 1, \ldots, 1\right) \in \mathbf{R}^{n}: \alpha \in \mathbf{N} \cup\{0\}\right\}$ for $n \geq 2$. Then we have $\operatorname{Ass}\left(\mathbf{U}_{1}^{-1} \mathbf{R}\right)=\{(0)\}=\partial \mathbf{F}_{\mathbf{R} 0} \cap \operatorname{Supp}\left(\mathbf{U}_{0}[1]^{-1} \mathbf{R}\right), \operatorname{Ass}\left(\mathbf{U}_{2}^{-2} \mathbf{R}\right)=\{(x)\}=\partial \mathbf{F}_{\mathbf{R} 1}$ $\cap \operatorname{Supp}\left(\mathbf{U}_{2}^{-2} \mathbf{R}\right)=\partial \mathbf{F}_{\mathbf{R} 1} \cap \operatorname{Ass}\left(\mathbf{U}_{1}[1]^{-2} \mathbf{R}\right)$ and $\mathbf{U}_{i}^{-i} \mathbf{R}=0$ for all $i \geq 3$. But $\mathbf{U}_{2}^{-2} \mathbf{R} \not\left(\mathbf{U}_{2}^{-2} \mathbf{R}\right)_{(x)}$.

Example (3.10). Let $\mathbf{R}=k[[X, Y, Z]]$ and $\mathbf{M}=k[[X, Y, Z]] /(X) \cap\left(X^{2}, Y\right)$ $=k[[x, y, z]]$. Let $\mathbf{U}_{1}=\left\{\left(Y^{n}\right) \in \mathbf{R}^{1}: n \geq 0\right\}$. Let $\mathbf{F}_{i}=\{\mathfrak{p} \in \operatorname{Spec}(\mathbf{R}):$ ht $\mathfrak{p} \geq$ 
$i+1\} \quad$ for $\quad i \geq 0$. Then $\operatorname{Ass}\left(\mathbf{U}_{1}^{-1} \mathbf{M}\right)=\{(X)\}=\partial \mathbf{F}_{0} \cap \operatorname{Ass}(\mathbf{M})=\partial \mathbf{F}_{0} \cap$ $\operatorname{Ass}\left(\mathbf{U}_{0}[1]^{-1} \mathbf{M}\right)$ but $\mathbf{M}_{Y} \cong \mathbf{U}_{1}^{-1} \mathbf{M} \not\left(\mathbf{U}_{1}^{-1} \mathbf{M}\right)_{(X)} \cong \mathbf{M}_{(X)}$.

\section{REFERENCES}

[B] N. Bourbaki, "Commutative algebra," Addison-Wesley publishing company, 1972.

[C] S. C. Chung, Associated prime ideals and isomorphisms of modules of generalized fractions, to appear in Math. J. Toyama Univ., 17 (1994).

[GO] G. J. Gibson and L. O'carroll, Direct limit systems, generalized fractions and complexes of Cousin type, J. Pure Appl. Algebra, 54 (1988), 249-259.

[HS] M. A. Hamieh and R. Y. Sharp, Krull dimension and generalized fractions, Proc. Edinburgh Math. Soc., 28 (1985), 349-353.

[M] H. Matsumuta, "Commutative ring theory," Cambridge University Press, 1986.

[O] L. O'carroll, On the generalized fractions of Sharp and Zakeri, J. London Math. Soc., (2) 28 (1983), 417-427.

[RSZ] A. M. Riley, R. Y. Sharp and H. Zakeri, Cousin complexes and generalized fractions, Glasgow Math. J., 26 (1985), 51-67.

[S1] R. Y. Sharp, The Cousin complex for a module over a commutative Noetherian ring, Math. Z., 112 (1969), 340-356.

[S2] - Gorenstein modules, Math. Z., 115 (1970), 117-139.

[S3] - On the structure of certain exact Cousin complexes, LN in Pure Appl. Math., 84 (1981), 275-290.

[S4] - A Cousin complex characterization of balanced big CohenMacaulay modules, Quart. J. Math. Oxford Ser., (2) 33 (1982), 471-485.

[ST] R. Y. Sharp and Z. Tang, On the structure of Cousin complexes, J. Math. Kyoto Univ., 33-1 (1993), 285-297.

[SY] R. Y. Sharp and M. Yassi, Generalized fractions and Hughes' grade-theoretic analogue of the Cousin complex, Glasgow Math. J., 32 (1990), 173-188.

[SZ1] R. Y. Sharp and H. Zakeri, Modules of generalized fractions, Mathematika, 29 (1982), 32-41.

[SZ2] - Modules of generalized fractions and balanced big Cohen-Macaulay modules, Commutative Algebra: Durham 1981, London Mathematical Society Lecture Notes, 72 (Cambridge University Press, 1982), 61-82.

[SZ3] - Local cohomology and modules of generalized fractions, Mathematika, 29 (1982), 296-306.

[SZ4] - Generalized fractions, Buchsbaum modules and generalized Cohen-Macaulay modules, Math. Proc. Camb. Phil. Soc., 98 (1985), 429-436.

[SV] J. Stückrad and W. Vogel, "Buchsbaum rings and applications," SpringerVerlag, 1986.

Department of Mathematics

School of Science

Nagoya University

Nagoya, 464-01, Japan
Current address:

Department of Mathematics

Chungnam National University

Taejon 305-764, Korea 